\title{
Dispersal and species' responses to climate change
}

\author{
Justin M. J. Travis, Maria Delgado, Greta Bocedi, Michel Baguette, Kamil Bartoń, \\ Dries Bonte, Isabelle Boulangeat, Jenny A. Hodgson, Alexander Kubisch, \\ Vincenzo Penteriani, Marjo Saastamoinen, Virginie M. Stevens and James M. Bullock
}

J. M. J. Travis (justin.travis@abdn.ac.uk) and G. Bocedi, School of Biological Sciences, Univ. of Aberdeen, Zoology Building, Tillydrone Avenue, Aberdeen, AB24 2TZ, UK. - M. Delgado and M. Saastamoinen, Dept of Biosciences, Univ. of Helsinki, Viikinkaari 1, FI-00014 Helsinki, Finland. - M. Baguette, Museum National d'Histoire Naturelle, Dept Ecologie et Gestion de la Biodiversité, and CNRS USR2936, Station d'Ecologie Expérimentale, route du CNRS, FR-09200 Moulis, France. - K. Bartoń and A. Kubisch, Field Station Fabrikschleichach, Univ. of Wuerzburg, Glashuettenstrasse 5, DE-96181 Rauhenebrach, Germany. - D. Bonte, Dept of Biology, Ghent Univ., K. L. Ledeganckstraat 35, BE-9000 Ghent, Belgium. - I. Boulangeat, Laboratoire d'Ecologie Alpine, UMR CNRS 5553, Univ. Joseph Fourier, BP 53, FR-38041 Grenoble Cedex 9, France. - J. A. Hodgson, Dept of Biology, Wentworth Way, Univ. of York, York, YO10 5DD, UK. - V. Penteriani, Dept of Conservation Biology, Estación Biológica de Doñana, CSIC, c/Américo Vespucio s/n, ES-41092 Seville, Spain. VP also at: Finn. Mus. of Nat. Hist., Zool. Mus. Pohjoinen Rautatiekatu 13, Univ. of Helsinki, FI-00100 Helsinki, Finland. - V. M. Stevens, CNRS USR2936, Station d'Ecologie Expérimentale, route du CNRS, FR-09200 Moulis, France. VMS also at: F.R.S.-FNRS, Unité de Biologie du Comportement, Univ. de Liège, 22 Quai van Beneden, BE-4020 Liège, Belgium. - J. M. Bullock, Centre for Ecology and Hydrology, Maclean Building, Benson Lane, Crowmarsh Gifford, Wallingford, OX10 8BB, UK.

\begin{abstract}
Dispersal is fundamental in determining biodiversity responses to rapid climate change, but recently acquired ecological and evolutionary knowledge is seldom accounted for in either predictive methods or conservation planning. We emphasise the accumulating evidence for direct and indirect impacts of climate change on dispersal. Additionally, evolutionary theory predicts increases in dispersal at expanding range margins, and this has been observed in a number of species. This multitude of ecological and evolutionary processes is likely to lead to complex responses of dispersal to climate change. As a result, improvement of models of species' range changes will require greater realism in the representation of dispersal. Placing dispersal at the heart of our thinking will facilitate development of conservation strategies that are resilient to climate change, including landscape management and assisted colonisation.
\end{abstract}

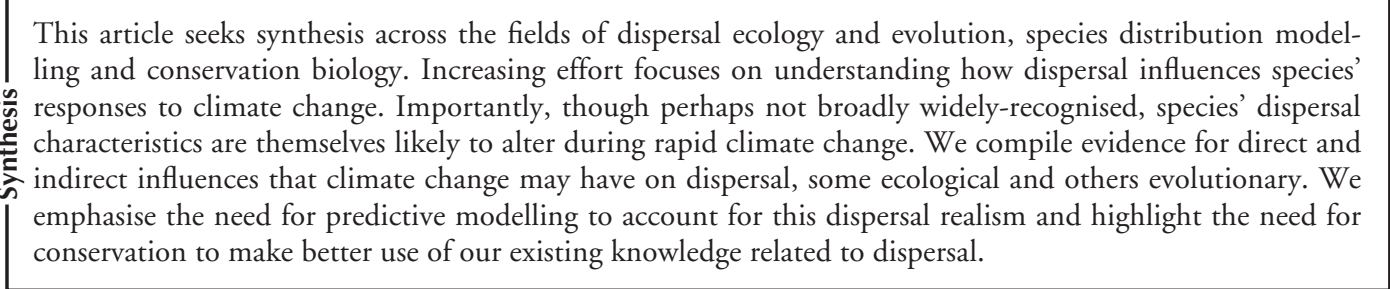

Ongoing rapid climate change is resulting in the geographic shifting of species' suitable environmental conditions (IPCC 2007, Chen et al. 2011). Species might survive this rapid change by shifting their distributions or through evolution such that populations become adapted to the new local climatic conditions (Berg et al. 2010, Bellard et al. 2012). For both these responses, dispersal is a central process; it determines the potential spread rate of a population and, as the process by which genes are moved between populations, it

The review and decision to publish this paper has been taken by the above noted SE. The decision by the handling SE is shared by a second SE and deputy EiC. influences the rate of adaptation to changing conditions and the potential for evolutionary rescue (Bell and Gonzalez 2011). Thus, understanding, predicting and managing biodiversity responses to rapid climate change demands a full consideration of species' dispersal characteristics and how these characteristics may themselves change under climate change. Despite this, the great majority of studies projecting future species' distributions do not explicitly account for dispersal (Thomas et al. 2004, Thuiller et al. 2006). A significant number of review and perspectives papers considering climate change and biodiversity responses have been published recently, covering both conceptual and methodological aspects of modelling range shifts (Thuiller et al. 2008, 
Elith and Leathwick 2009, Dawson et al. 2011, McMahon et al. 2011, Bellard et al. 2012, Bocedi et al. 2012, Schurr et al. 2012). Although these reviews tackle the issue of how dispersal is important in governing responses to climate change, importantly they do not examine the role of climate change-driven changes in dispersal.

Here, we demonstrate that climate change is likely to have direct and indirect impacts on the dispersal of individuals, and will exert new selection pressures leading to dispersal evolution. We argue for the incorporation of the emerging synthesis concerning the ecology and evolution of dispersal into models of species' spread under climate change, with an explicit consideration of the resulting uncertainties. Moreover, we stress that the development of climate change resilient conservation strategies has seldom benefited from the improved ecological and evolutionary knowledge about dispersal, and believe that including dispersal details will help resolve current heated debates about future conservation strategies (Hodgson et al. 2009, Doerr et al. 2011, Thomas 2011, Webber et al. 2011). The conceptual scope of this perspectives article is therefore purposely broad, covering a number of topics such as observed ecological and evolutionary patterns, theory, models and conservation.

\section{Will climate change reduce or enhance individual dispersal abilities?}

The initiation of dispersal (emigration) by an individual, its subsequent movements (transfer) and its settlement decisions (immigration), are influenced by local conditions, and so climate change may affect an individual's dispersal. Predicted impacts of climate change on means and variabilities of temperatures, rainfall, storm events, wind speed, snow and ice cover, $\mathrm{CO}_{2}$ concentrations, etc. (IPCC 2007) could affect the dispersal process directly, and also indirectly by changing the biophysical environment (e.g. habitat quality, availability of food resources, etc.) and the state of individuals (body size and morphology, body condition and rate of development). Table 1 summarises empirical evidence for such effects among different taxa (including vascular plants, algae, insects, reptiles, birds, fish and mammals) in both terrestrial and aquatic (marine and continental) systems. The decision to disperse can be affected directly by changes in temperature (Battisti et al. 2006, Pärn et al. 2011, Delattre et al. 2013), in windspeed (Thomas et al. 2003), in storms (Lea et al. 2009), in flooding (Roche et al. 2012), and in snow cover (Schwartz et al. 2009). Changes in climatic factors can also have a direct impact on organisms during the transfer phase of dispersal, either by increasing (Dickison et al. 1986, Censky et al. 1998, Peirson et al. 2008, Kuparinen et al. 2009, Cormont et al. 2011, Monzón-Argüello et al. 2012) or decreasing the dispersal distance (Geffen et al. 2007, Bullock et al. 2012). In one recent case that highlights a further potential complexity, the impact of temperature on dispersal distance was shown to interact with the degree of habitat fragmentation (Delattre et al. 2013): dispersal distance was greater at lower temperatures in fragmented landscape while, in more continuous landscapes, dispersal distance was greater under warmer conditions.

Multiple indirect effects of climate change on dispersal are also possible. For example, smaller individuals often have reduced dispersal ability, and climate-induced deterioration in habitat quality or faster development during higher temperatures can both reduce body size in a variety of species (Gibbs et al. 2011, McCauley and Mabry 2011). However, poor conditions in the biophysical environment can also increase the probability of emigration in some other species (Figuerola 2007). Species which rely on other biota for dispersal, such as seeds carried by ants, will suffer if the phenology of the dispersal agent becomes asynchronous under climate change (Warren et al. 2011). The phenology of American redstart on breeding grounds shows a climate-driven latitudinal gradient, such that natal dispersal distances decrease when the growing season starts earlier (Studds et al. 2008).

Our survey indicates multiple and varied climate impacts on dispersal and that the predicted changes in specific climatic factors may both increase and decrease dispersal abilities depending on the system and species considered (Table 1). For example, non-optimal temperatures may induce flotation behaviour and thus dispersal in aquatic molluscs (Correia Rosa et al. 2012), but can decrease the probability of dispersal in spiders (Bonte et al. 2008) and lizards (Massot et al. 2008). In addition, opposing consequences may arise in a single species. For example, dispersal of wind-dispersed thistles should decrease with predicted reductions in wind speed (Bullock et al. 2012), but should increase as plants grow taller in warmer conditions (Zhang et al. 2011).

Such variation in the impact of climate change on dispersal will become better understood and more predictable as dispersal mechanisms themselves are better elucidated and incorporated into dispersal models - see below. Climate change will also affect other aspects of life history such as fecundity and mortality, which will determine the effectiveness of dispersal at a population level. For example, if climate change results in a local population having a higher fecundity it may indirectly increase the number of individuals dispersing, while if there is higher mortality in new habitat colonised at the range edge, it will decrease the number of effective colonists.

\section{How will dispersal evolve under climate change?}

A classic study related the dispersal characteristics of lodgepole pine seeds to its post-glacial spread (Cwynar and MacDonald 1987); seeds in populations towards the still expanding colonisation front were more dispersive than those in longer established populations. We now have accumulating evidence of similar increases in dispersal ability in populations shifting their ranges in response to contemporary environmental change. For example, at expanding range margins the frequency of long-winged morphs of bush crickets is increased (Simmons and Thomas 2004) and speckled wood butterflies invest more in thorax size, which increases their flight power (Hill et al. 1999).

These observations of increased dispersal at expanding margins conform to theoretical expectations. Models have demonstrated that, at expanding margins, selection can: favour increased emigration rates, even when local populations are at low densities (Travis et al. 2009); promote risky movement behaviours, enhancing the possibility of long distance dispersal even if mortality is increased 


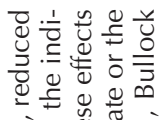

可记

흥 है है 울

ㄴ.

प)

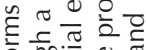

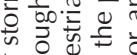

论

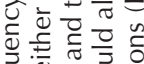

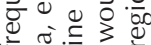

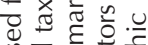

过

등응

के है

ช.

㐘

능 त क क

E

๘

.0.0

ड़े

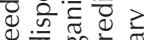

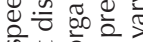

웅

उत

zे 는

우의

ฐ

을

눈

过

记

는

品

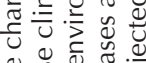

ڤั)

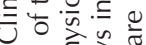

可证 응

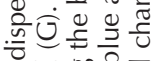

중

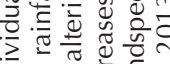

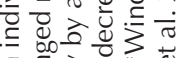

ธ。 㛡

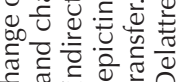

Q

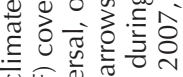

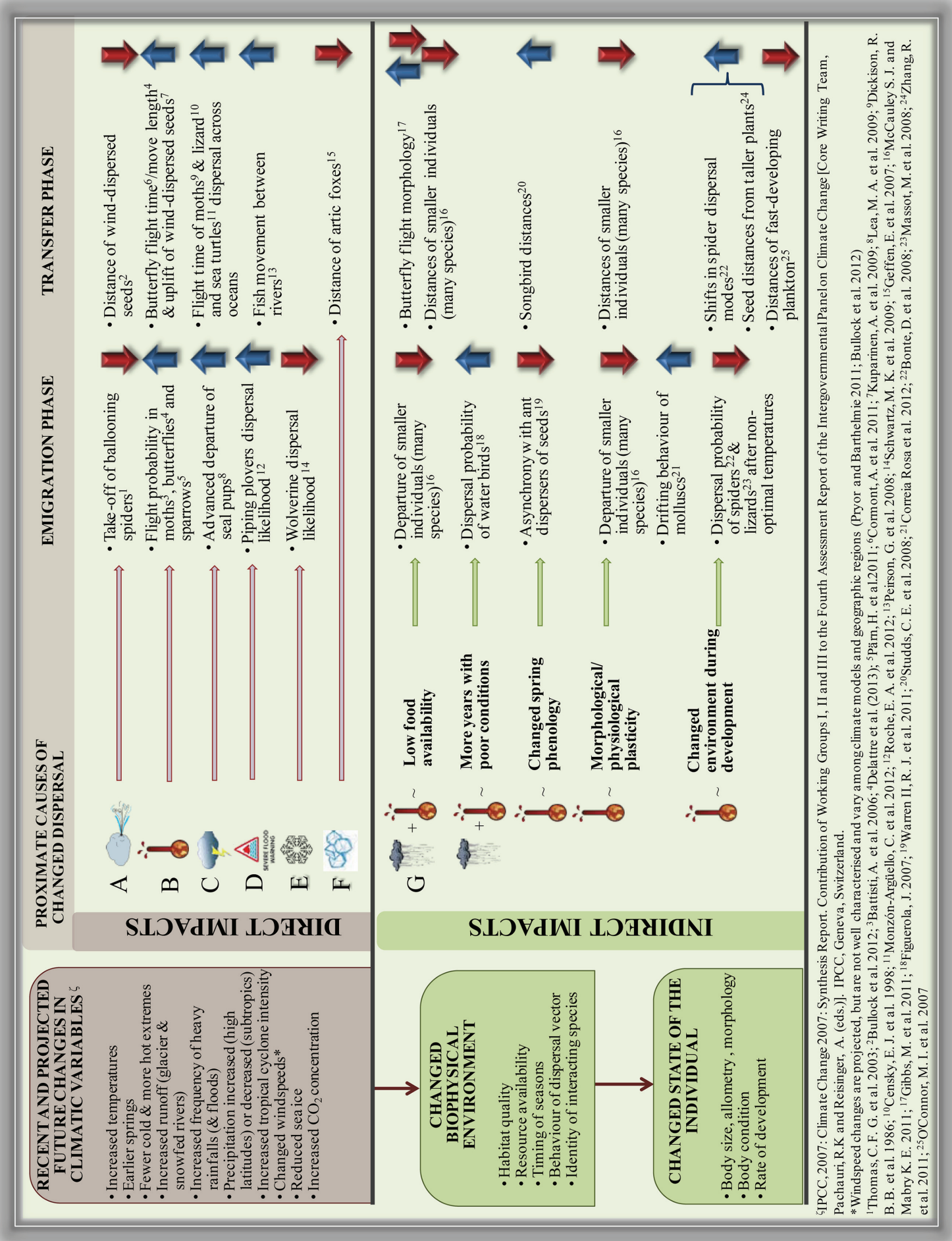

tᄂ

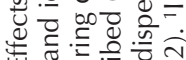

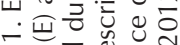

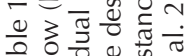

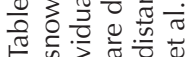


(Bartoń et al. 2012); and foster investment in dispersal traits at the expense of other life-history attributes (Burton et al. 2010). While the same selective forces that act on dispersal in stationary ranges, including kin competition and inbreeding depression, may still play a role at expanding margins, selection will now favour dispersal strategies which maximise the likelihood that some descendants follow the expanding margin (Travis et al. 2009, Bartoń et al. 2012, Henry et al. 2013).

Most theory exploring dispersal evolution during range expansions has assumed that species spread across homogeneous landscapes (Travis et al. 2009, Burton et al. 2010). While this provides some generic insights, it is clearly not realistic for most biogeographic ranges. Recent modelling has begun to shed light on how dispersal will evolve, and influence the spatial structure of species' ranges, when range expansions occur across environmental gradients (Kubisch et al. 2010, Phillips 2011). The evolution of increased dispersal at expanding range margins may have unexpected consequences in heterogeneous landscapes (Travis et al. 2010, Phillips 2011). It can allow a species to cross large gaps between habitat patches, and this is more likely when the population has been expanding for long enough to have evolved greater dispersal ability (Travis et al. 2010). Counter-intuitively, evolutionary rescue might be promoted under increased rates of experienced climate change (Boeye et al. 2013). However, increased dispersal at expanding range margins may not always result in species spreading more readily across heterogeneous landscapes, as it can diminish the ability to adapt to local environmental conditions and ultimately lead to reduced spread rate (Phillips 2011). These initial theoretical studies serve to highlight the likely complexity of eco-evolutionary dispersal dynamics at expanding range margins. Increased eco-evolutionary understanding and subsequently improved prediction of biogeographic range-shifting requires integration of ecology and evolution.

Whether dispersal is evolving in reality, or if organisms show phenotypic plasticity is a challenging question due to the complex and poorly understood interactions between plastic and genetic processes. Yet, the difference is important for understanding the current and likely future consequences of climate change, because it informs us about the extent to which populations are subject to natural selection resulting from a changing environment (Visser 2008). Recent studies present evidence that we can expect global warming to impact species in both ways (Balanyá et al. 2006, Bradshaw and Holzapfel 2006, Charmantier et al. 2008). However, while the evidence for climate change driven phenotypic responses in wild populations is strong, empirical data allowing us to evaluate whether or not any genetic response - and hence evolution - has actually occurred are still rather scarce (Bradshaw and Holzapfel 2006, Reusch and Wood 2007, Gienapp et al. 2008). Indeed, it is still questionable whether for most organisms evolution can be rapid enough to allow adaptation to changes in climatic conditions, especially as evidence suggest that adaptation is favoured under gradual environmental change (Bell and Gonzalez 2011) and that it can impose demographic costs (Lynch and Lande 1993, Bürger and Lynch 1995). The impact of a given rate of climate change may differ between species due to disparate generation times, and this, together with variability in standing genetic variation within populations, is likely to determine their potential for local adaptation (Berg et al. 2010). Phenotypic plasticity can thus play a central role by providing the potential for organisms to respond rapidly and effectively to environmental change. Ultimately, changes due to phenotypic plasticity may be advantageous because it is possible that a changing environment may select for differing patterns of plasticity among individuals (i.e. plasticity itself can evolve by natural selection; Sultan et al. 2012). However, many species may lack both the plasticity that would allow them to better cope with climate change and the genetic variation that would allow them to evolve in response to climate change. Their long generation times and relatively small population sizes make evolutionary adaptation unlikely. And it is unclear if they have enough phenotypic plasticity to successfully adapt to new climate conditions.

\section{How is dispersal included in predicting species' future distributions?}

The last decade has seen a massive effort in developing species' distribution models (SDMs) to project where species are likely to be found under future scenarios of environmental change (Thomas et al. 2004, Thuiller et al. 2006). The most widely used approach relates species' location data to environmental variables including climate. By using the outputs of global climate models, these so-called habitat suitability models can project species distributions onto future climatic conditions. Amongst the well-understood limitations of this approach (Franklin 2010, Dawson et al. 2011), the complete lack of, or incomplete treatment of dispersal is key (Fig. 1). Indeed, this lack is also apparent in more mechanistic approaches, which use an understanding of species' climatic tolerances to construct process-based distribution models (Dormann et al. 2012).

An initial method used to establish bounds of uncertainty in species' range changes has been to run models assuming that species exhibit either unlimited or no dispersal. Such studies demonstrate huge differences between the two scenarios in projections of, for example, regional species' extinctions (Thomas et al. 2004) and functional diversity of trees across Europe (Thuiller et al. 2006). The resulting appreciation of the importance of dispersal has stimulated a recent move towards more integrated models that seek to account for dispersal, population dynamics and habitat dynamics, as well as climate and habitat suitability (Franklin 2010).

Recent studies have proposed hybrid models that couple habitat suitability models with more mechanistic models accounting for dispersal in a variety of ways (Fig. 1). A simple way to incorporate dispersal has been to couple habitat suitability models with colonisation models that are based on nearest-neighbour dispersal whereby landscape grid cells that become climatically suitable can be colonised if a neighbouring cell is already occupied (Midgley et al. 2006, RouraPascual et al. 2009, Willis et al. 2009). A more sophisticated and increasingly used approach for including dispersal in predictive species' distribution models is to fit a statistical function (i.e. dispersal kernels) to observed dispersal data (Pagel and Schurr 2012, Schurr et al. 2012). Dispersal kernels 


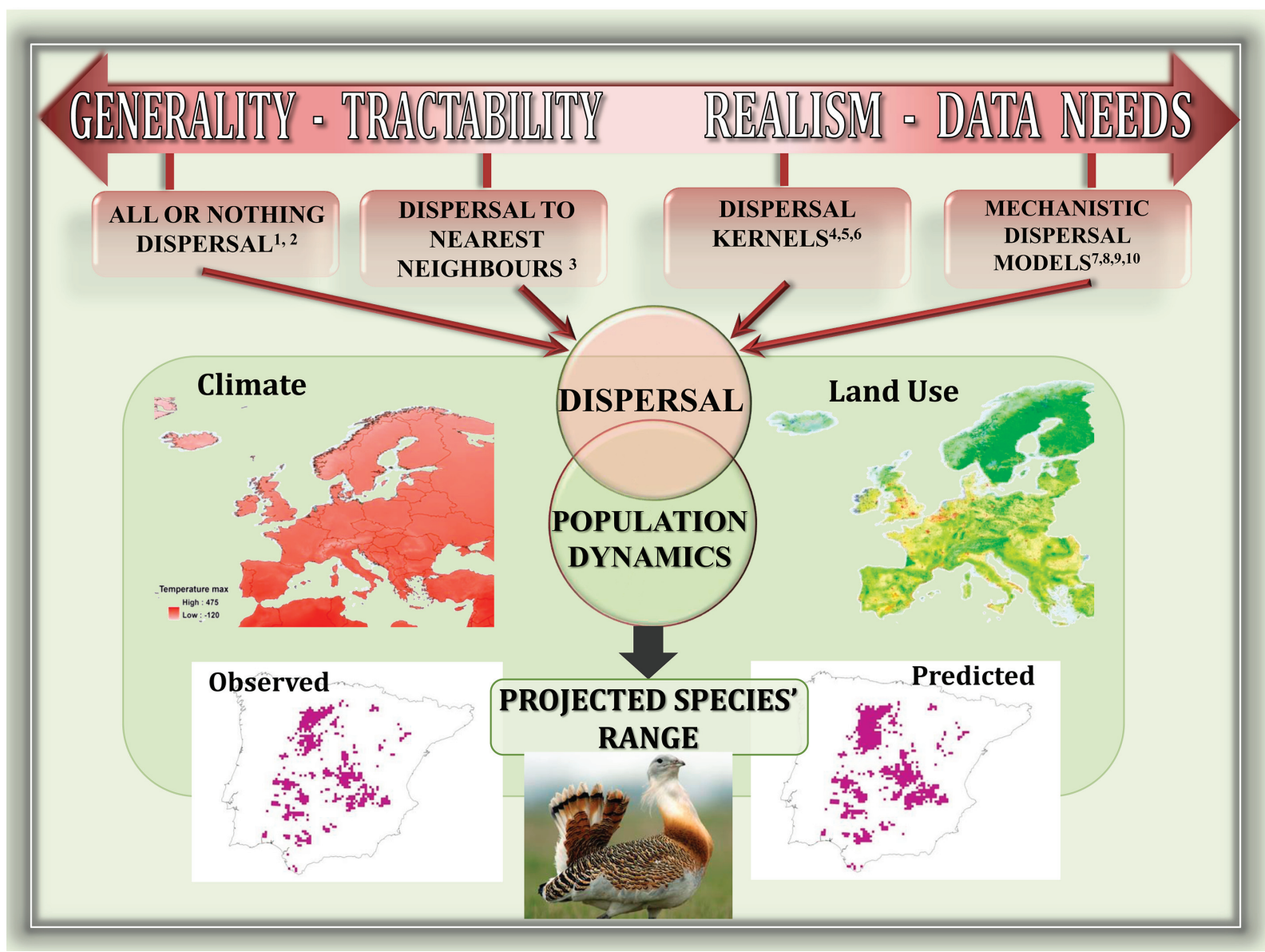

Figure 1. Dispersal will be the heart of a new generation of process-based models developed to predict, and inform the management of, species' responses to environmental change. By incorporating dispersal together with an explicit representation of population dynamics, models will become much better able to simulate the spatio-temporal dynamics of species under alternative future climate and land-use scenarios. To date, most projections of biodiversity responses to climate change have been made using all or nothing dispersal with fewer examples of nearest-neighbour dispersal or statistical dispersal kernels. While more detailed mechanistic dispersal models have been developed both for animal and plant dispersal, they have yet to be used extensively in the climate change field. In part this is due to the substantial challenges faced with these more sophisticated models, both in terms of the data needs for parameterisation and the greater computation needs of these more complex approaches. We argue that incorporating greater realism in the dispersal process will result in improved predictive capability, particularly when there are likely to be synergistic impacts of climate and land use change. ${ }^{1}$ Thomas et al. $2004,{ }^{2}$ Thuiller et al. 2006, ${ }^{3}$ Pitt et al. 2009, ${ }^{4}$ Iverson et al. 2011, ${ }^{5}$ Carey 1996, ${ }^{6}$ Engler and Guisan 2009, ${ }^{7}$ Bullock et al. 2012, ${ }^{8} \mathrm{Nathan}$ et al. $2011,{ }^{9} \mathrm{McRae}$ et al. 2008, ${ }^{10}$ Travis et al. 2012.

have already been integrated with habitat suitability models in few studies, through coupling with a simple migration model (Engler et al. 2009) or spatially explicit metapopulation models (RAMAS-GIS; Keith et al. 2008, Anderson et al. 2009). This last kind of hybrid model offers the possibility to consider not only the transfer (as dispersal distance) phase but also the emigration and immigration ones by incorporating the population dynamics. Yet, the major challenge of this method is to represent the influential, but rare, long distance dispersal events that are often poorly described by standard statistical distributions. One way of achieving this is to use a mixture of two statistical distributions, obtaining fat tail distributions that include long distance dispersal events (Engler et al. 2009, Pagel and Schurr 2012).

Because different internal (e.g. individual condition, sex) and external (e.g. the local environment) factors can alter individual dispersal processes (Clobert et al. 2009), the distribution of dispersal distances is unlikely to be a fixed property of a species. Including this complexity requires models that represent the dispersal process more mechanistically, allowing dispersal distances to vary in space and time. For instance, a mechanistic model of seed dispersal by wind, based on a mathematical simplification of a complex stochastic model of seed transport in turbulent air (Katul et al. 2005), has been applied to project the spread rates of plant populations under a changing climate (Nathan et al. 2011, Bullock et al. 2012). Similarly, simple animal movement trajectories across a landscape have been simulated with individuals also able to select an optimal patch from all those within a maximum dispersal radius (McRae et al. 2008). This shift towards mechanistic modelling offers the opportunity to incorporate potentially crucial details related to the ecology and evolution of dispersal and promises to yield new tools that can inform the development of improved conservation strategies for a changing environment. 


\section{How do conservation strategies for climate change account for dispersal?}

Following the emergence of island biogeography theory in the 1960 s and then even more since the rise to prominence of the metapopulation concept in the 1990s, conservation biologists and managers have increasingly recognised the importance of dispersal in their decision making. Climate change has forced a further shift towards dynamic, spatial considerations, at larger scales than considered previously. Recognition that contemporary conservation needs to facilitate the shifting of species' biogeographic ranges and promote local adaptation has resulted in a number of potential interventions being suggested, including landscape management, assisted colonisation and genetic reinforcement (assisted adaptation) (Loss et al. 2011). It is agreed that landscape management is essential to facilitate population spread, although there is considerable discussion regarding the optimal balance between managing for habitat area and quality and managing elements of the landscape for connectivity between habitat patches (Hodgson et al. 2009, 2011, Doerr et al. 2011). Most connectivity models consider spatial dispersal processes as a simple function of distance instead of considering the dynamics of emigration, movement between patches and settlement decisions that together result in colonisation.

There is also a heated debate about the relative merits and dangers of the more interventionist approaches. One school of thought is that the risk of species' extinctions resulting from inactivity demands that we attempt assisted colonisation (Thomas 2011), whilst the other highlights problems concerning the feasibility and the potential damaging ecological effects of translocation (Webber et al. 2011). While this debate continues, assisted colonisation is rapidly gaining credibility among conservation practitioners (HoeghGuldberg et al. 2008, Richardson et al. 2009, Minteer and Collins 2010, Vitt et al. 2010, Loss et al. 2011), yet we currently lack any underpinning theory related to how, if we attempt assisted colonisation, we should best proceed.

\section{Outlook: improving predictive tools and adapting conservation strategies}

An increased understanding of dispersal under climate change is critical to inform the deployment of effective climate change resilient conservation strategies. We have shown that much is already known about the complexity of dispersal in terms of both ecology and evolution. We believe that representing some of this complexity in models can help to improve their utility, especially in applying them to novel climates and environments. Dispersal-informed modelling must become a central tool for planning conservation strategies that combine elements of both assisted colonisation and spatial planning and which exploit species' eco-evolutionary dispersal dynamics to maximise survival under climate change. Here we suggest five priority areas for research over the coming years.

First, while considerable knowledge related to the ecology and evolution of dispersal has already been gained, there remain critical gaps that empirical work should address urgently. The development of protocols to gather high-reso- lution data at different scales - from local, small scale studies and on manipulative experiments to global environmental or geographical gradients - will certainly help modellers to implement such data in a systematic manner, assuring comparability of model-based results and, therefore, improving forecasting on how biodiversity will respond to ongoing global climate change (Nogués-Bravo and Rahbek 2011). It is timely that several trait databases that are gathered all over the world (for plants see Kattge et al. 2011) will be available soon. Strategic use of meta-analyses on these datasets (Stevens et al. 2012) will help to reveal the patterns and causes of heterogeneity in dispersal between and within species. By offering the opportunity to apply complex dispersal model to numerous species, these datasets will be crucial for explaining biodiversity patterns by a mechanistic evaluation of processes across different spatial and temporal scales (Nogués-Bravo and Rahbek 2011).

Second, future modelling should represent dispersal more realistically, taking advantage of the considerable knowledge we already have on the three phases of dispersal, emigration, transfer and settlement (Bonte et al. 2012, Travis et al. 2012), and how these are affected by the biotic and abiotic environmental context. For example, we anticipate that models including density-dependent emigration are likely to yield more robust results than existing density-independent models. We believe that including mechanistic movement models for the transfer phase is crucial (Dullinger et al. 2012). This will allow better prediction of dispersal in heterogeneous landscapes and under the novel alterations in the biophysical environment caused by climate change. Taking the mechanistic approach and using maps of, for example, varying wind speeds or densities of animal dispersal vectors, will result in a species' dispersal varying according to local biotic and abiotic context. Importantly, a mechanistic approach also reduces the requirement for direct measurements of the rare long distance dispersal events that have a disproportionate impact on rates of spread (Neubert and Caswell 2000, Clark et al. 2001). Movement modelling has progressed rapidly over the last decade, driven by the development of new technologies and statistical methods that together enable efficient collection and analysis of movement data (Morales et al. 2010). The challenge now is to link these movement models to population dynamics (Morales et al. 2010, Nathan et al. 2011, Bullock et al. 2012, Travis et al. 2012).

Third, while there is strong evidence for substantial within-species variability in dispersal and evolution during range expansions, predicitive models have yet to address this complexity (Hoffmann and Sgrò 2011). Recently, evolution of egg desiccation resistance has been incorporated in a biophysical model to predict climate impacts on the range of the dengue fever vector Aedes aegypti (Kearney et al. 2009). Similar approaches for dispersal are needed and, ultimately, it would be desirable to have a suite of models that couple dispersal evolution with local adaptation. Importantly, by incorporating this complexity we will be better placed to determine the uncertainty associated with predictions. Embracing the uncertainty will result in a shift from presenting binary outcomes (species presence or absence) towards a probabilistic prediction of future range limits (Pagel and Schurr 2012).

Fourth, there is a need to understand how evolutionary responses to climate change will vary among taxa. Indeed, 
not all species will show selection for increased dispersal under climate change. Before we are able to incorporate evolution of dispersal realistically into predictive models, we need to gain increased empirical knowledge of the genetic factors that may hinder or facilitate these adaptive changes. In particular, we need to improve understanding on how genetic covariances among dispersal and other fitness-related life history traits will influence the rate and direction of dispersal evolution. Dispersal and its evolution are strongly integrated with particular demographic, behavioural, physiological or morphological traits into life histories (Burton et al. 2010, Bonte et al. 2012, Stevens et al. 2012), and we may expect that the association between dispersal and suites of traits will vary among species and whether selection occurred before or after dispersal (Scheiner et al. 2012). As a result, tradeoffs may constrain selection for increased dispersal in spreading populations (Burton et al. 2010). Finally, to assess whether observed responses to climate change correspond to genetic or phenotypic changes, and predict which species can adapt to a changing climate, more data on the strength of selection, heritability, as well as the development of quantitative genetic methods are needed (Gienapp et al. 2008). Although it will clearly be challenging to develop species-specific models to predict the role dispersal evolution will play in biogeographic range shifts, we are optimistic that the first attempts at doing so will emerge in the next five years. The challenge then will be generalising beyond the small number of species for which sufficient data exists to parameterise this degree of complexity to a broad suite of species. Stevens et al. (2013) recently demonstrated that a species' dispersal characteristics can be predicted quite successfully through correlation with a set of life history traits. As information on dispersal lability becomes available for some species, it may be possible to extend this approach to infer the evolvability of dispersal in other species. A further potential approach to gain information on the likely rate of dispersal evolution might be to utilise palaeoecological data to infer how fast dispersal evolved in species during past periods of biogeographical shifts.

Fifth, the planning of assisted dispersal to facilitate both colonisation and adaptation requires consideration, through modelling, of the locations of source and recipient populations, and the timing of translocation (Weeks et al. 2011). The optimal approach is likely to depend on the ecology and evolution of a species' dispersal strategy. Spread beyond the area of introduction will be governed by habitat configuration and the organisms' dispersal abilities (Hodgson et al. 2012). In fact, landscape management, of both habitat and non-habitat, could be made increasingly effective if we account for the different phases of the dispersal process. For example, large and high quality habitat patches will maintain sustainable populations, and are easily located by dispersers. However, spread rates may be enhanced by creating smaller and moderate quality patches as these will promote density-dependent emigration (Travis et al. 2009). Management activities that reduce the energetic, mortality and time costs of the transfer phase through non-habitat can increase both the net displacement of individuals and the fitness of individuals at their final destination (Bonte et al. 2012). Finally, effective settlement can be enhanced through the main- tenance of high quality recipient habitat (Edelaar et al. 2008, Edelaar and Bolnick 2012).

The sourcing of individuals for translocation should consider the substantial intra-specific variability in dispersal ability (Bonte et al. 2012, Stevens et al. 2012). Taking individuals from populations where selection has already increased dispersal should enhance spread. However, given the likely tradeoffs with other life history traits, these more dispersive individuals may have reduced probability of successful establishment. High probability of colonisation, establishment of persistent populations and rapid spread, may be accomplished by introducing a mix of individuals with contrasted strategies. This 'composite provenancing' will also increase the adaptive potential of the new populations, by maximizing standing genetic variation for a range of life-history traits that will together determine the viability of local populations and the rate of population spread across a landscape (Weeks et al. 2011).

In conclusion, interventions seem essential to avoid species extinctions under climate change. However, to make such actions fruitful, new understanding about the evolution and ecology of dispersal must be taken into account. Some species may show such limited dispersal ability, under current and future scenarios (Bullock et al. 2012), that any but the most extreme interventions will be doomed to failure. In contrast, given the evidence of species evolving dispersal during range expansions, we may not need to intervene for all species that initially show no range shifting - they just need time to evolve.

Acknowledgements - JMJT and MD has contributed equally and thus share first authorship. We thank T. Benton, T. Hovestadt, P. Jordano, O. Ovaskainen, J. Reid as well as Univ. of Aberdeen 4th year Advanced Behavioural Ecology and Wildlife Management Classes for comments, and S. Ojanen and M. Triviño for helping with the images of Fig. 1. JMJT, MB, KB and VMS were funded by TenLamas, GB, MB and JMB by SCALES, and IB by ECOCHANGE (all EU projects). MD (no. 140367) and MS (no. 132697) received post-doctoral grants from the Academy of Finland. DB is funded by FWO project G.0610.11 and FWO Research Network EVENET (with JMJT), and VP by the Junta of Andalucía (Excellence Project, RNM-5090). JMJT is funded by NERC. MB and VMS are supported by TULIP (ANR-10LABX-41).

\section{References}

Anderson, B. J. et al. 2009. Dynamics of range margins for metapopulations under climate change. - Proc. R. Soc. B 276: 1415-1420.

Balanyá, J. et al. 2006. Global genetic change tracks global climate warming in Drosophila subobscura. - Science 313: 1773-1775.

Bartoń, K. A. et al. 2012. Risky movement increases the rate of range expansion. - Proc. R. Soc. B 279: 1194-1202.

Battisti, A. et al. 2006. A rapid altitudinal range expansion in the pine processionary moth produced by the 2003 climatic anomaly. - Global Change Biol. 12: 662-671.

Bell, G. and Gonzalez, A. 2011. Adaptation and evolutionary rescue in metapopulations experiencing environmental deterioration. - Science 332: 1327-1330.

Bellard, C. et al. 2012. Impacts of climate change on the future of biodiversity. - Ecol. Lett. 15: 365-377. 
Berg, M. P. et al. 2010. Adapt or disperse: understanding species persistence in a changing world. - Global Change Biol. 16: 587-598.

Bocedi, G. et al. 2012. Projecting species' range expansion dynamics: sources of systematic biases when scaling up patterns and processes. - Meth. Ecol. 3: 1008-1018.

Boeye, J. et al. 2013. More rapid climate change promotes evolutionary rescue through selection for increased dispersal distance. - Evol. Appl. 6: 353-364.

Bonte, D. et al. 2008. Thermal conditions during juvenile development affect adult dispersal in a spider. - Proc. Natl Acad. Sci. USA 105: 17000-17005.

Bonte, D. et al. 2012. Costs of dispersal. - Biol. Rev. Camb. Phil. Soc. 87: 290-312.

Bradshaw, W. E. and Holzapfel, C. M. 2006. Evolutionary response to rapid climate change. - Science 312: 1477-1478.

Bullock, J. M. et al. 2012. Modelling spread of British winddispersed plants under future wind speeds in a changing climate. - J. Ecol. 100: 104-115.

Burton, O. J. et al. 2010. Tradeoffs and the evolution of life-histories during range expansion. - Ecol. Lett. 13: 1210-1220.

Bürger, R. and Lynch, M. 1995. Evolution and extinction in a changing environment: a quantitative-genetic analysis. Evolution 49: 151-163.

Carey, P. D. 1996. DISPERSE: a cellular automaton for predicting the distribution of species in a changed climate. - Global Ecol. Biogeogr. 5: 217-226.

Censky, E. J. et al. 1998. Over-water dispersal of lizards due to hurricanes. - Nature 395: 556.

Charmantier, A. et al. 2008. Adaptive phenotypic plasticity in response to climate change in a wild bird population. - Science 320: 800-803.

Chen, I.-C. et al. 2011. Rapid range shifts of species associated with high levels of climate warming. - Science 333: 1024-1026.

Clark, J. S. et al. 2001. Invasion by extremes: population spread with variation in dispersal and reproduction. - Am. Nat. 157: 537-554.

Clobert, J. et al. 2009. Informed dispersal, heterogeneity in animal dispersal syndromes and the dynamics of spatially structured populations. - Ecol. Lett. 12: 197-209.

Cormont, A. et al. 2011. Effect of local weather on butterfly flight behaviour, movement and colonization: significance for dispersal under climate change. - Biodivers. Conserv. 20: 483-503.

Correia Rosa, I. et al. 2012. Effects of upper-limit water temperatures on the dispersal of the Asian clam Corbicula fluminea. - PloS ONE 7: e46635.

Cwynar, L. C. and MacDonald, G. M. 1987. Geographical variation of lodgepole pine in relation to population history. - Am. Nat. 129: 463-469.

Dawson, T. P. et al. 2011. Beyond predictions: biodiversity conservation in a changing climate. - Science 332: 53-58.

Delattre, T. et al. 2013. Interactive effects of landscape and weather on dispersal. - Oikos 122: 1576-1585.

Dickison, R. B. B. et al. 1986. Spruce budworm moth flight and storms: further studies using aircraft and radar. - J. Clim. Appl. Meteorol. 25: 1600-1608.

Doerr, V. A. J. et al. 2011. Connectivity, dispersal behaviour, and conservation under climate change: a response to Hodgson et al. - J. Appl. Ecol. 48: 143-147.

Dormann, C. F. et al. 2012. Correlation and process in species distribution models: bridging a dichotomy. - J. Biogeogr. 39: 2119-2131.

Dullinger, S. et al. 2012. Extinction debt of high-mountain plants under 21st-century climate warming. - Nature Climate Change 2: 619-622.

Edelaar, P. and Bolnick, D. I. 2012. Non-random gene flow: an underappreciated force in evolution and ecology. - Trends Ecol. Evol. 27: 659-665.
Edelaar, P. et al. 2008. Matching habitat choice causes directed gene flow: a neglected dimension in evolution and ecology. - Evolution 62: 2462-2472.

Elith, J. and Leathwick, J. R. 2009. Species distribution models: Ecological explanation and prediction across space and time. - Annu. Rev. Ecol. Evol. Syst. 40: 677-697.

Engler, R. et al. 2009. Predicting plant distribution and dispersal in a changing climate. - Divers. Distrib. 15: 590-601.

Figuerola, J. 2007. Climate and dispersal: black-winged stilts disperse further in dry springs. - PLoS ONE 2: e539.

Franklin, J. 2010. Moving beyond static species distribution models in support of conservation biogeography. - Divers. Distrib. 16: 321-330.

Geffen, E. et al. 2007. Sea ice occurrence predicts genetic isolation in the Arctic fox. - Mol. Ecol. 16: 4241-4255.

Gibbs, M. et al. 2011. Phenotypic plasticity in butterfly morphology in response to weather conditions during development. - J. Zool. 283: 162-168.

Gienapp, P. et al. 2008. Climate change and evolution: disentangling environmental and genetic responses. - Mol. Ecol. 17: $167-178$.

Henry, R. C. et al. 2013. Eco-evolutionary dynamics of range shifts: elastic margins and critical thresholds. - J. Theor. Biol. 321: $1-7$.

Hodgson, J. A. et al. 2009. Climate change, connectivity and conservation decision making: back to basics. - J. Appl. Ecol. 46: 964-969.

Hodgson, J. A. et al. 2011. Habitat re-creation strategies for promoting adaptation of species to climate change. - Conserv. Lett. 4: 289-297.

Hodgson, J. A. et al. 2012. The speed of range shifts in fragmented landscapes. - PloS ONE 7: e47141.

Hill, J. K. et al. 1999. Evolution of flight morphology in a butterfly that has recently expanded its geographic range. - Oecologia 121: 165-170.

Hoegh-Guldberg, O. et al. 2008. Assisted colonization and rapid climate change. - Science 321: 345-346.

Hoffmann, A. A. and Sgrò, C. M. 2011. Climate change and evolutionary adaptation. - Nature 470: 479-485.

Iverson, L. R. et al. 2011. Lessons learned while integrating habitat, dispersal, disturbance, and life-history traits into species habitat models under climate change. - Ecosystems 14: 1005-1020.

IPCC 2007. Climate change 2007: Synthesis report. Contrib. Working Grp I, II and III to the 4th Assess. Rep. Intergovernmental Panel on Climate Change. Pachauri, R. K. and Reisinger, A. (eds). - IPCC, Geneva, Switzerland.

Kattge, J. et al. 2011. TRY - a global database of plant traits. - Global Change Biol. 17: 2905-2935.

Katul, G. G. et al. 2005. Mechanistic analytical models for long-distance seed dispersal by wind. - Am. Nat. 166: 368-381.

Kearney, M. et al. 2009. Integrating biophysical models and evolutionary theory to predict climatic impacts on species' ranges: the dengue mosquito Aedes aegypti in Australia. - Funct. Ecol. 23: 528-538.

Keith, D. A. et al. 2008. Predicting extinction risks under climate change: coupling stochastic population models with dynamic bioclimatic habitat models. - Biol. Lett. 4: 560-563.

Kubisch, A. et al. 2010. On the elasticity of range limits during periods of expansion. - Ecology 91: 3092-3099.

Kuparinen, A. et al. 2009. Increases in air temperature can promote wind-driven dispersal and spread of plants. - Proc. R. Soc. B 276: 3081-3087.

Lea, M. A. et al. 2009. Extreme weather events influence dispersal of naive northern fur seals. - Biol. Lett. 5: 252-257.

Loss, S. R. et al. 2011. Assisted colonization: integrating conservation strategies in the face of climate change. - Biol. Conserv. 144: $92-100$. 
Lynch, M. and Lande, R. 1993. Evolution and extinction in response to environmental change. - In: Kareiva, P. M. et al. (eds), Biotic interactions and global change. Sinauer, pp. 234-250.

Massot, M. et al. 2008. Climate warming, dispersal inhibition and extinction risk. - Global Change Biol. 14: 461-469.

McCauley, S. J. and Mabry, K. E. 2011. Climate change, body size, and phenotype dependent dispersal. - Trends Ecol. Evol. 26: 554-555.

McMahon, S. M. et al. 2011. Improving assessment and modelling of climate change impacts on global terrestrial biodiversity. - Trends Ecol. Evol. 26: 249-259.

McRae, B. H. et al. 2008. A multi-model framework for simulating wildlife population response to land-use and climate change. - Ecol. Modell. 219: 77-91.

Midgley, G. F. et al. 2006. Migration rate limitations on climate change-induced range shifts in Cape Proteaceae. - Divers. Distrib. 12: 555-562.

Minteer, B. A. and Collins, J. P. 2010. Move it or lose it? The ecological ethics of relocating species under climate change. - Ecol. Appl. 20: 1801-1804.

Monzón-Argüello, C. et al. 2012. Lost at sea: genetic, oceanographic and meteorological evidence for storm-forced dispersal. - J. R. Soc. Interface 9: 1725-1732.

Morales, J. M. et al. 2010. Building the bridge between animal movement and population dynamics. - Phil. Trans. R. Soc. B 365: 2289-2301.

Nathan, R. et al. 2011. Spread of North American wind-dispersed trees in future environments. - Ecol. Lett. 14: 211-219.

Neubert, M. G. and Caswell, H. 2000. Demography and dispersal: calculation and sensitivity analysis of invasion speed for structured populations. - Ecology 81: 1613-1628.

Nogués-Bravo, D. and Rahbek, C. 2011. Communities under climate change. - Science 334: 1070.

O'Connor, M. I. et al. 2007. Temperature control of larval dispersal and the implications for marine ecology, evolution and conservation. - Proc. Natl Acad. Sci. USA 104: 1266-1271.

Pagel, J. and Schurr, F. M. 2012. Forecasting species ranges by statistical estimation of ecological niches and spatial population dynamics. - Global Ecol. Biogeogr. 21: 293-304.

Pärn, H. et al. 2011. Spatial heterogeneity in the effects of climate and density-dependence on dispersal in a house sparrow metapopulation. - Proc. R. Soc. B 279: 144-152.

Peirson, G. et al. 2008. Lateral dispersal and displacement of fish during flood events in lowland river systems in the UK-implications for sustainable floodplain management. - Ecohydrol. Hydrobiol. 8: 363-373.

Phillips, B. L. 2011. Range shift promotes the formation of stable range edges. - J. Biogeogr. 39: 153-161.

Pitt, J. P. W. et al. 2009. Predicting Argentine ant spread over the heterogeneous landscape using a spatially explicit stochastic model. - Ecol. Appl. 19: 1176-1186.

Pryor, S. C. and Barthelmie, R. J. 2011. Assessing climate change impacts on the near-term stability of the wind energy resource over the United States. - Proc. Natl Acad. Sci. USA 108: 8167-8171.

Reusch, T. B. and Wood, T. E. 2007. Molecular ecology of global change. - Mol. Ecol. 16: 3973-3992.

Richardson, D. M. et al. 2009. Multidimensional evaluation of managed relocation. - Proc. Natl Acad. Sci. USA 106: 9721-9724.

Roche, E. A. et al. 2012. Flooding affects dispersal decisions in piping plovers (Charadrius melodus) in prairie Canada. - Auk 129: 296-306.

Roura-Pascual, N. et al. 2009. Consensual predictions of potential distributional areas for invasive species: a case study of
Argentine ants in the Iberian Peninsula. - Biol. Invas. 11: 1017-1031.

Scheiner, S. M. et al. 2012. The genetics of phenotypic plasticity. XI. Joint evolution of plasticity and dispersal rate. - Ecol. Evol. 2: 2027-2039.

Schurr, F. M. et al. 2012. How to understand species' niches and range dynamics: a demographic research agenda for biogeography. - J. Biogeogr. 39: 2146-2162.

Schwartz, M. K. et al. 2009. Wolverine gene flow across a narrow climatic niche. - Ecology 90: 3222-3232.

Simmons, A. D. and Thomas, C. D. 2004. Changes in dispersal during species' range expansions. - Am. Nat. 164: 378-395.

Stevens, V. M. et al. 2012. How is dispersal integrated in life histories: a quantitative analysis using butterflies. - Ecol. Lett. 15: 74-86.

Stevens, V. M. et al. 2013. Dispersal syndromes and the use of life-histories to predict dispersal. - Evol. Appl. 6: 630-642.

Studds, C. E. et al. 2008. Natal dispersal driven by environmental conditions interacting across the annual cycle of a migratory songbird. - Proc. Natl Acad. Sci. USA 105: 2929-2933.

Sultan, S. et al. 2012. A resurrection study reveals rapid adaptive evolution within populations of an invasive plant. - Evol. Appl. 6: 266-278.

Thomas, C. D. 2011. Translocation of species, climate change, and the end of trying to recreate past ecological communities. - Trends Ecol. Evol. 26: 216-221.

Thomas, C. F. G. et al. 2003. Aerial activity of linyphiid spiders: modelling dispersal distances from meteorology and behaviour. - J. Appl. Ecol. 40: 912-927.

Thomas, C. D. et al. 2004. Extinction risk from climate change. - Nature 427: 145-148.

Thuiller, W. et al. 2006. Using niche-based modelling to assess the impact of climate change on tree functional diversity in Europe. - Divers. Distrib. 12: 49-60.

Thuiller, W. et al. 2008. Predicting global change impacts on plant species' distribution: future challenges. - Persp. Plant Ecol. Evol. Syst. 9: 137-152.

Travis, J. M. J. et al. 2009. Accelerating invasion rates result from the evolution of density-dependent dispersal. - J. Theor. Biol. 259: 151-158.

Travis, J. M. J. et al. 2010. Towards a mechanistic understanding of dispersal evolution in plants: conservation implications. - Divers. Distrib. 16: 690-702.

Travis, J. M. J. et al. 2012. Modelling dispersal: an eco-evolutionary framework incorporating emigration, movement, settlement behaviour and the multiple costs involved. - Meth. Ecol. Evol. 3: 628-641.

Visser, M. E. 2008. Keeping up with a warming world; assessing the rate of adaptation to climate change. - Proc. R. Soc. B 275: 649-659.

Vitt, P. et al. 2010. Assisted migration of plants: changes in latitudes, changes in attitudes. - Biol. Conserv. 143: 18-27.

Warren II, R. J. et al. 2011. Temperature cues phenological synchrony in ant-mediated seed dispersal. - Global Change Biol. 17: 2444-2454.

Webber, B. L. et al. 2011. Translocation or bust! A new acclimatization agenda for the 21st century? - Trends Ecol. Evol. 26: 495-496.

Weeks, A. R. et al. 2011. Assessing the benefits and risks of translocations in changing environments: a genetic perspective. - Evol. Appl. 4: 709-725.

Willis, S. G. et al. 2009. Dynamic distribution modelling: predicting the present from the past. - Ecography 32: 5-12.

Zhang, R. et al. 2011. Warming increases the spread of an invasive thistle. - PLoS ONE 6: e21725. 